\title{
Les chefs d'établissements allemands face à une gouvernance nouvelle : acteurs ou objets ?
}

\section{Stefan Brauckmann et Denise Klinge}

Traducteur : Adeline Hurmaci

\section{OpenEdition Journals}

Édition électronique

URL : https://journals.openedition.org/ries/5585

DOI : 10.4000/ries.5585

ISSN : 2261-4265

Éditeur

France Education international

Édition imprimée

Date de publication : 1 décembre 2016

Pagination : $30-35$

ISBN : 978-2-85420-612-8

ISSN : $1254-4590$

\section{Référence électronique}

Stefan Brauckmann et Denise Klinge, «Les chefs d'établissements allemands face à une gouvernance nouvelle : acteurs ou objets ? », Revue internationale d'éducation de Sèvres [En ligne], 73 | décembre 2016, mis en ligne le 01 décembre 2018, consulté le 02 juillet 2021. URL : http:// journals.openedition.org/ries/5585; DOI : https://doi.org/10.4000/ries.5585

Ce document a été généré automatiquement le 2 juillet 2021.

(c) Tous droits réservés 


\title{
Les chefs d'établissements allemands face à une gouvernance nouvelle : acteurs ou objets?
}

\author{
Stefan Brauckmann et Denise Klinge \\ Traduction : Adeline Hurmaci
}

\section{Introduction}

1 Dans le contexte des résultats des enquêtes comparatives internationales sur les performances qui s'inscrivent dans le modèle paradigmatique de développement et d'efficience scolaires, les Länder allemands, avec le passage d'un pilotage par les «inputs» à un pilotage par les «outputs» fondé sur l'évaluation des résultats, s'engagent actuellement dans un processus de modernisation du système scolaire (Brauckmann et al., 2011). Le législateur a juridiquement tenu compte du fait qu'un rôle clé revient au chef d'établissement dans la garantie et le développement de la qualité (Wissinger, 2011). Ainsi au cours des 20 dernières années les Länder allemands ont-ils, à des degrés divers, accordé davantage d'autonomie aux établissements et, dans cette perspective, renforcé le statut et la position de leurs responsables. Ceux-ci, portant l'entière responsabilité de leur établissement, sont l'interface entre les environnements scolaire et extra-scolaire.

Des fondements empiriques de plus en plus nombreux attestant du rôle central de l'action de direction dans la garantie et le développement de la qualité de l'établissement ont contribué dans de nombreux pays, européens ou non, à l'élaboration de stratégies de recrutement, de sélection, de préparation, de formation et de soutien pour les chefs d'établissement. Ces stratégies de développement présupposent que les chefs d'établissement acquièrent de nouveaux concepts susceptibles d'influer sur l'orientation de leur activité.

3 La recherche internationale sait relativement peu de l'usage, de l'impact (Bessoth, 2005) et des conditions structurelles d'encadrement de programmes de 
professionnalisation « à succès ». Ces derniers revendiquent en particulier l'adéquation entre les besoins personnels (par exemple, la motivation) et les conditions générales structurelles (offres d'accompagnement dans le choix d'une mesure), ainsi que la liaison entre une professionnalisation réalisée par un prestataire externe et une autoinitiation. Pourtant, la professionnalisation est, d'une manière générale, considérée comme ayant une influence positive sur les connaissances, les compétences et le comportement des participants.

4 Si la professionnalisation de l'action de direction est de plus en plus revendiquée, on s'aperçoit rapidement que peu d'études se préoccupent de savoir quelles sont les conditions systémiques, organisationnelles et individuelles, dont l'action de direction et le ressenti en matière de charge de travail dépendent. Jusqu'ici, seuls quelques modèles se proposent de prendre en compte les facteurs potentiels influençant l'action de direction dans les établissements scolaires. On observe deux desiderata fondamentaux dans la recherche scientifique : d'une part, la tentative de séparer, à un niveau empirique, le contexte institutionnel et l'organisation des acteurs, et d'autre part, celle de modéliser, de manière empirique satisfaisante, les relations évoquées.

\section{Protocole d'enquête de l'étude SHaRP}

5 Dans ce contexte, le projet de recherche «L'action des chefs d'établissements entre droits et devoirs élargis » (SHaRP), soutenu par le ministère fédéral de l'éducation et de la recherche, vise à une explication empiriquement fondée de la manière dont les chefs d'établissements vivent leur charge de travail dans le contexte des nouvelles conceptions de pilotage (Brauckmann, 2014).

6 L'intérêt de la recherche se porte sur l'évaluation de leur temps de travail et les conditions contextuelles de leur action sur fond d'exigences devenant toujours plus complexes. On suppose que les nouvelles lignes directrices en matière de gestion conduisent à la confrontation, non seulement pour l'établissement mais aussi, et en particulier, pour son responsable, avec des missions transformées et de nouveaux défis qui se répercutent sur leur profil d'activité et de charge.

7 Dans le cadre de cette recherche, il est particulièrement intéressant de voir la manière dont les chefs d'établissement considèrent et interprètent leurs activités dans les champs d'action que sont la conception et l'administration. Il est également intéressant de voir les contradictions constitutives auxquelles ils ont affaire dans l'exercice de leur nouveau rôle au sein de l'organisation scolaire (orientation collective, modèles sociaux d'interprétation).

\section{Analyses et commentaires}

\section{Une multitude de nouveautés dans la politique d'enseignement}

8 Ce qui est considéré comme problématique par l'ensemble des chefs d'établissement interviewés, c'est que l'on attende d'eux qu'ils appliquent en même temps toutes les nouvelles réglementations, alors qu'elles sont multiples. La chance de mettre en place un procédé structuré serait ainsi compromise et les processus pédagogiques seraient inutilement interrompus. Plus d'un chef d'établissement se voit ainsi appelé, dans son 
rôle de responsable, à éviter d'éventuelles défaillances au niveau du développement de son école ou lycée, quitte à intervenir directement si nécessaire. Le directeur ou proviseur, particulièrement engagé dans l'application de l'innovation en matière de politique d'enseignement, est toujours contraint d'expliquer tout changement de règlement, tout retard ou toute modification auprès de ses collègues. Des directives systémiques, sous forme d'innovation en matière de politique d'enseignement, caractérisées par des césures et des discontinuités ou s'avérant inconsistantes d'un point de vue conceptuel, peuvent nuire, le cas échéant, à l'« autorité " du chef d'établissement, si elles génèrent des incohérences dans le déroulement organisationnel. Ces dernières peuvent en effet lui être reprochées dans son rôle en tant qu'intermédiaire et promoteur de la réforme, en tant que bras droit de l'administration scolaire ${ }^{1}$.

\section{Une plus grande autonomie des établissements, une obligation plus forte à rendre compte}

9 Les chefs d'établissement interviewés ont un avis mitigé sur la procédure «nouveau pilotage ». Leur motivation diffère quant à l'ambition de tester et contribuer à la conception des chemins menant à l'autonomie. Ils gèrent de manière très différente les nouvelles missions et nouveaux défis de l'autonomie scolaire. Les données des entretiens indiquent que le degré de motivation des directeurs et proviseurs correspond généralement à l'attitude de leurs collègues face à la nouvelle philosophie de pilotage et à la disposition au changement de l'environnement scolaire. Le souhait d'une plus grande autonomie n'est pas aussi ferme partout. Cela est notamment dû aux exigences administratives, qui doivent être remplies en parallèle du processus de transformation en vue d'une école autonome, et qui rendent ainsi difficiles les activités qui y sont liées. L'investissement administratif, par exemple, pour des mesures de formation continue que le chef d'établissement devrait effectuer, serait bien trop important par rapport à ce qu'il apporterait. L'investissement bureaucratique pour les établissements scolaires serait tout simplement trop élevé et les procédures administratives prendraient trop de temps. Il faudrait que les chefs d'établissements soient déchargés des fonctions administratives avec, par exemple, la mise en place d'un personnel supplémentaire dans ce domaine, tel un assistant d'administration scolaire.

10 Ce qui est représenté de manière particulièrement négative, ce sont les expériences au cours desquelles de nouvelles dispositions juridiques viennent contraindre le travail pédagogique. Dans l'ensemble, les chefs d'établissement, et en particulier dans le Land de Brandebourg, ont l'impression que de nouvelles ordonnances et de nouveaux décrets d'application contribuent à la réduction de libertés qui étaient jusqu'alors garanties. Sur ce point, des convictions pédagogiques sont parfois sensiblement opposées aux nouvelles réglementations juridiques. Le vécu ou ressenti individuel d'une plus grande autonomie (dans le sens où le chef d'établissement dispose de plus grandes marges de manœuvre dans la conception) n'est pas nécessairement dû à de " nouvelles " prescriptions juridiques ; il dépend également fortement de la disposition au développement des collègues sur place. De la même façon, cette disposition peut être le résultat d'un bouleversement historique (contexte propre à l'ensemble de la société) tel que la chute du mur (l'« après-tournant $\left.»^{2}\right)$. 
11 Les jugements positifs des nouveaux concepts de pilotage et du nouveau profil de mission des chefs d'établissement se réfèrent en majeure partie à la budgétisation des frais de personnel qui permet de mieux réagir face à l'annulation potentielle de cours.

En ce qui concerne le recrutement et le développement professionnel du personnel scolaire, l'image qui se dessine est plus nuancée. Ici, les jugements portés sur les réglementations liées à l'autonomie de l'établissement et relatives au personnel sont extrêmement variés. Une partie des interviewés s'exprime de manière très positive sur les possibilités réelles de recrutement. En revanche, si l'on considère la gestion des ressources humaines, certains évoquent les mesures de développement du personnel. Celles-ci sont considérées comme problématiques, car elles ne peuvent être intégrées sans césure dans le concept d'une plus grande autonomie. Ainsi, les chefs d'établissements seraient parfois amenés à préparer une décision pour l'administration scolaire, sans eux-mêmes pouvoir répondre ou justifier de leur décision face aux candidats entrant en ligne de compte et qui font partie de leur corps enseignant.

13 Si les données qualitatives font ressortir certaines prises de position face au « nouveau pilotage ", il est très rare que soient évoquées des réussites au niveau d'un établissement, qui pourraient être mises en relation avec les nouvelles lignes directrices. Or, c'est seulement de cette manière que les concepts peuvent devenir partie intégrante de structures existantes. En revanche, les chefs d'établissement se sentent majoritairement plus autonomes dans leur rôle d'enseignant que dans celui de cadre dirigeant.

On remarque un certain scepticisme quant à la fonction de contrôles de qualité centralisés ${ }^{3}$. D'après les directeurs et proviseurs, ceux-ci ne contribueraient pas à l'amélioration de la qualité des établissements. Ce sont principalement les proviseurs de lycée qui évoquent ce thème. L'obligation à rendre compte est surtout destinée au personnel d'établissement et s'appuie sur une série d'instruments internes d'évaluation. Il en ressort donc que les chefs d'établissement se sentent essentiellement obligés de rendre compte auprès de la commission de leur établissement. De même, les résultats d'évaluations externes sont principalement exploités et analysés en interne.

\section{Collaboration avec l'inspection et l'administration centrale ${ }^{4}$}

Les données montrent une tension entre autodétermination scolaire et devoir de légitimation vers l'extérieur. Dans ce contexte, la collaboration avec l'inspection se présente comme particulièrement difficile. Un déséquilibre se dessine dans la façon de concevoir l'obligation à rendre compte et la responsabilité de direction (disposition à l'obligation de rendre compte). Ainsi, l'inspection demanderait des renseignements détaillés dans de courts laps de temps, alors qu'elle-même n'est pas toujours en mesure d'informer le personnel.

En outre, inspection et chef d'établissement ne disposeraient par exemple pas toujours d'informations équivalentes sur l'établissement concerné, ce qui rendrait une coordination régulière difficile. La question qui se pose ici est notamment de savoir avec quelle efficacité il est possible de saisir non seulement le travail de l'établissement, mais aussi celui de l'administration scolaire. De plus, une importante fluctuation au niveau du personnel de cette administration rendrait difficile la communication sur le stade et les objectifs primaires de développement de l'établissement. En outre, d'après 
les chefs d'établissement interviewés, des formulaires uniformes seraient souhaitables pour permette une gestion flexible et simplifiée des missions administratives.

\section{Dispositifs de soutien et de consultation}

17 Les chefs d'établissement interviewés ont des opinions divergentes quant à savoir si la formation continue doit se faire en fonction du type d'établissement, ou bien si elle doit être adaptée à chaque établissement dans sa spécificité. Souvent, les chefs d'établissements plus petits peuvent s'approprier les valeurs empiriques d'établissements plus grands. D'autres, en revanche, insistent sur le fait que le contenu de la formation continue s'oriente de façon unidimensionnelle vers les problèmes et les spécificités du type d'établissement qui est majoritairement représenté dans le groupe de formation.

18 Alors que les chefs d'établissement s'expriment parfois de manière très critique sur les formations continues proposées par les instituts respectifs des Länder, les formations continues financées par le fond budgétaire propre et réalisées par des coachs extrascolaires connaissent une plus large satisfaction.

19 À en juger la manière dont les chefs d'établissement conçoivent leur fonction de direction, il faudrait que des ressources humaines (secrétariat) et matérielles (équipement technique) soient disponibles pour qu'ils puissent se familiariser avec leur rôle en tant que cadre dirigeant.

\section{Du créateur à l'administrateur}

20 L'une des personnes interviewées formule une critique sur la profusion de concepts managériaux sans cohérence d'ensemble. Les missions de développement dégénèrent ainsi en une série formelle d'exercices administratifs imposés qui surchargent l'activité des directeurs d'établissement, sans pour autant mener à un résultat visible ou présentable.

\section{Synthèse}

21 Les directeurs et proviseurs interviewés dans cette étude partielle qualitative ne semblent pas encore avoir réfléchi - ou du moins pas de manière approfondie - aux nouvelles lignes de pilotage d'établissement scolaire autonome et aux conséquences qui en découlent pour leur action. C'est plutôt la prudence qui domine dans les entretiens, révélant que la nouvelle fonction de direction n'est pas encore assez bien établie. Plusieurs interviewés indiquent être au début de processus de développement qui se doivent d'être observés. Un sentiment de confusion s'exprime néanmoins face à l'imbrication croissante des activités qui comporte un risque d'incohérence. De plus, des activités invisibles se multiplieraient parallèlement au développement des activités prescrites. C'est probablement la raison pour laquelle il a été difficile pour l'ensemble de nos interviewés - à une exception près - de rendre compte de leurs activités. De même, les responsables d'établissement ont longuement hésité avant de classer les temps de travail hebdomadaires, d'autant qu'il leur a fallu prendre en compte le caractère fréquemment saisonnier de certaines activités, telles que la préparation aux examens de fin d'années, celle des bulletins scolaires, etc. 
Les déclarations des personnes interrogées laissent supposer que, dans le contexte du «nouveau pilotage », il n'existe pas de conception claire du rôle de chacun dans la direction d'établissement, ou du moins qu'elle est perçue comme incomplète ou opaque. Ainsi, une grande partie des interviewés disent ne pas constater de changement dans leur attitude envers leurs collègues enseignants dans les champs d'action du "nouveau pilotage». Il est donc presque impossible de trouver des indications ou descriptions qui rendraient compte, de manière précise, des conséquences concrètes des nouvelles lignes de pilotage sur l'activité professionnelle du chef d'établissement, sauf si celui-ci a déjà été sensibilisé dans sa formation de directeur ou proviseur aux « nouveaux » champs d'action.

Ils exercent plutôt un « leadership vers l'intérieur », en portant leur regard analytique sur les conceptions d'objectifs et de développement pour l'organisation et la réalisation de l'enseignement qui sont individuelles ou bien spécifiques à un établissement, à un domaine ou à un groupe de matières. Les problèmes qui surviennent en classe, dans le cadre du travail avec les élèves, sont importants aux yeux des chefs d'établissement, car ils perturbent régulièrement l'organisation scolaire et déterminent de manière essentielle la culture pédagogique d'un établissement.

Dans l'ensemble, les chefs d'établissement insistent sur certaines activités qui s'opèrent par petites étapes et dont il n'est pas possible - ou difficilement- d'estimer l'« impact ». Ils portent une attention particulière aux aspects contraignants du travail quotidien opérationnel. En outre, les interviews révèlent que les chefs d'établissement jugent différemment ce qu'ils doivent faire, ce qui influe sur la disposition à déléguer. La tentative de créer des marges de manœuvres pédagogiques provoque parfois des déviations dans l'organisation et la conception de cours (curriculum interne à l'établissement, variations dans les emplois du temps).

\section{BIBLIOGRAPHIE}

BESSOTH R. (2005) : « Für was sollen Schulleitungen qualifiziert werden? » Zeitschrift für Schulleitung und Schulberatung, 16 (3), S. 104-107.

BRAUCKMANN S. (2014) : « Zwischen erweiterten Rechten und Pflichten Empirische Befunde aus der 6-Länder-Schulleitungsstudie ShaRP », Schulmanagement, 3, S. 22-24.

BRAUCKMANN S., KÜHNE S., STÄSCHE U., TARAZONA M., WEISHAUPT H. \& WITTMANN E. (2010) : «Steuerung und Verwaltung des Bildungswesens ", in JÄGER R. S., NENNIGER P., PETILLON H., SCHWARZ B. \& WOLF B. (Hrsg.), Empirische Pädagogik 1990-2010, Bd 1: Grundlegende empirische pädagogische Forschung Landau: Verl. Empirische Pädagogik (S. 119-160).

WISSINGER J. (2011): « Schulleitung und Schulleitungshandeln », in TERHART E., BENNEWITZ H. \& ROTHLAND M. (Hrsg.), Handbuch der Forschung zum Lehrerberuf, Münster: Waxmann (S. 98-115). 


\section{NOTES DE FIN}

1. Schulverwaltung : équivalent du rectorat français en matière d'administration scolaire.

2. La Wende est désignée en français comme «le tournant», c'est pourquoi nous traduisons Nachwendezeit par « après-tournant ». (Ndt)

3. En allemand : zentrale Anschlussprüfungen.

4. Schulträger : unités administratives qui, à différents niveaux selon les Länder, assurent des fonctions de gestion des moyens et des ressources humaines et ont également une obligation de contrôle de qualité.

\section{INDEX}

Index géographique : Allemagne

Mots-clés : chef d'établissement, politique éducative

Palabras claves : director de escuela, política educacional

Keywords : school head, educational policy

\section{AUTEURS}

\section{STEFAN BRAUCKMANN}

Stefan Brauckmann est professeur des Universités et titulaire de la chaire sur le développement de la qualité et l'assurance qualité en éducation à l'Institute of instructional and school development (IUS) de l'Université Alpen-Adria à Klagenfurt, Autriche. Après un doctorat obtenu à l'Université libre de Berlin, il a travaillé comme chercheur au Centre de recherche sur la gouvernance éducative de l'Institut allemand pour la recherche internationale en éducation (DIPF), de l'association Leibniz (Berlin). Ses principaux centres de recherche et d'intérêt portent sur les différents mécanismes à l'œuvre dans l'administration de l'éducation, ainsi que sur l'impact du management et du leadership sur le développement de l'assurance qualité et des apprentissages dans le domaine de l'éducation. Courriel : Stefan.Brauckmann@aau.at

\section{DENISE KLINGE}

Denise Klinge a étudié les sciences de l'éducation à l'Université libre de Berlin, puis a travaillé comme chercheuse à l'Université de Potsdam et à l'Institut allemand de recherche pédagogique Internationale, où elle a soutenu une thèse de doctorat sur la prise de décision des parents allemands dans le parcours scolaire de leurs enfants. Actuellement, elle travaille à l'Université de la Bundeswehr à Munich sur le thème de la mesure de soi (« Quantified Self ») et sur les pratiques liées au développement de programmes et de techniques dans ce contexte. Courriel : denise.klinge@unibw.de 